\title{
SPECTROGRAMS AND TIME-FREQUENCY LOCALIZED FUNCTIONS IN THE HANKEL SETTING
}

\author{
Saifallah Ghobber, Siwar Hkimi And Slim OMri
}

\begin{abstract}
The uncertainty principle in Fourier analysis sets a limit to the possible simultaneous concentration of a function and its Hankel transform. Nevertheless, signals that have highly concentrated time-frequency content have many applications in quantum mechanics, PDE, engineering and in signal analysis. We use here time-frequency localization operators in the Hankel setting to measure the time-frequency content of functions on a subset of finite measure $\Sigma$ within the time-frequency plane. Then, using eigenfunctions and eigenvalues of these operators, we prove a characterization of functions that are time-frequency concentrated in $\Sigma$, and we obtain approximation inequalities for such functions using a finite linear combination of eigenfunctions, since they are maximally time-frequency-concentrated in the region of interest.
\end{abstract}

Mathematics subject classification (2010): 94A12, 45P05, 42C25, 42C40.

Keywords and phrases: Time-frequency concentration, localized functions, windowed Hankel transform, localization operator, spectrogram.

\section{REFERENCES}

[1] L. D. Abreu, K. Gröchenig, And J. L. Romero, On accumulated spectrograms, Trans. Amer. Math. Soc. 368 (2016), 3629-3649.

[2] L. D. Abreu And J. M. Pereira, Measures of localization and quantitative Nyquist densities, Appl. Comput. Harmon. Anal. 38 (2015), 524-534.

[3] C. BACCAR, N. B. HAMADI AND H. HERCH, Time-frequency analysis of localization operators associated to the windowed Hankel transform, Integral Transforms Spec. Funct. 27 (2016), 245-258.

[4] F. A. BEREZIN, Wick and anti-Wick operator symbols, Math. USSR-Sb. 151971.

[5] P. C. BowIE, Uncertainty inequalities for Hankel transforms, SIAM J. Math. Anal. 2 (1971), 601606.

[6] A. Cordoba And C. FefFerman, Wave packets and Fourier integral operators, Comm. Partial Differential Equations, 3 (1978), 979-1005.

[7] W. Czaja And G. Gigante, Continuous Gabor transform for strong hypergroups, J. Fourier Anal. Appl. 9 (2003), 321-339.

[8] I. DAUBECHIES, Time-frequency localization operators: a geometric phase space approach, IEEE Trans. Inform. Theory, 34 (1988), 605-612.

[9] G. A. M. Velasco AND M. DöRFLER, Sampling time-frequency localized functions and constructing localized time-frequency frames, European J. Appl. Math. 28 (2017), 854-876.

[10] S. GhobBer AND P. Jaming, Strong annihilating pairs for the Fourier-Bessel transform, J. Math. Anal. Appl. 377 (2011), 501-515.

[11] S. GHOBber AND P. JAMING, The Logvinenko-Sereda theorem for the Fourier-Bessel transform, Integral Transforms Spec. Funct. 24 (2013), 470-484.

[12] S. Ghobber And S. OMri, Time-frequency concentration of the windowed Hankel transform, Integral Transforms Spec. Funct. 25 (2014), 481-496.

[13] S. GhoвbeR, Phase space localization of orthonormal sequences in $L_{\alpha}^{2}\left(\mathbb{R}_{+}\right)$, J. Approx. Theory, 189 (2015), 123-136.

[14] S. Ghobвer, A variant of the Hankel multiplier, Banach J. Math. Anal. 12 (2018), 144-166.

[15] S. Ghobber, A dispersion inequality in the Hankel setting, Czech. Math. J. 68 (2018), 227-241. 
[16] B. M. Levitan, Series expansion in Bessel functions and Fourier integrals, Uspekhi Mat. Nauk. 6 (1951), 102-143.

[17] H. J. LANDAU, On Szegö's eigenvalue distribution theorem and non-Hermitian kernels, J. Anal. Math. 28 (1975), 335-357.

[18] H. J. Landau ANd H. O. Pollack, Prolate spheroidal wavefunctions, fourier analysis and uncertainty II, Bell Syst. Tech. J. 40 (1961), 65-84.

[19] H. J. LANDAU AND H. O. Pollack, Prolate spheroidal wavefunctions, fourier analysis and uncertainty III: The dimension of the space of essentially time- and band-limited signals, Bell Syst. Tech. J. 41 (1962), 1295-1336.

[20] T. Moumni, On essentially time and Hankel band-limited functions, Integral Transforms Spec. Funct. 23 (2012), 83-95.

[21] J. Ramanathan And P. TOPIWALA, Time-frequency localization and the spectrogram, Appl. Comput. Harmon. Anal. 1 (1994), 209-215.

[22] F. Riesz And B. Sz.-Nagy, Functional Analysis, Frederick Ungar Publishing Co., New York, 1955.

[23] H. Raunut, Time Frequency and Wavelet Analysis of Functions with Symmetry Properties, Logos Verlag Berlin, 2005.

[24] H. RAUHUT, Banach frames in coorbit spaces consisting of elements which are invariant under symmetry groups, Appl. Comput. Harmon. Anal. 18 (2005), 94-122.

[25] H. RAUhUT AND M. Rösler, Radial multiresolution in dimension three, Constr. Approx. 2 (2005), 193-218.

[26] D. SLePian AND O. Pollack, Prolate spheroidal wavefunctions, fourier analysis and uncertainty I, Bell Syst. Tech. J. 40 (1961), 43-63.

[27] M. W. WonG, Wavelet transforms and localization operators, Birkhäuser, 2002.

[28] A. ZAYED, Solution of the energy concentration problem in reproducing-kernel Hilbert space, SIAM J. Appl. Math. 75 (2015), 21-37. 